African Crop Science Journal by African Crop Science Society is licensed under a Creative Commons Attribution 3.0 Uganda License. Based on a work at www.ajol.info/ and www.bioline.org.br/cs DOI: http://dx.doi.org/10.4314/acsj.v24i3.6

\title{
SUSCEPTIBILITY TO BRUCHIDS AMONG COMMON BEANS IN UGANDA
}

\author{
J.A. EBINU ${ }^{1}$, V. NSABIYERA ${ }^{1,2}$, M. OTIM ${ }^{3}$, S.T. NKALUBO ${ }^{3}$, M.UGEN ${ }^{3}$, A.J. AGONA ${ }^{4}$ \\ and H.L. TALWANA ${ }^{1}$ \\ ${ }^{1}$ School of Agricultural Sciences College of Agricultural and Environmental Sciences, Makerere University, \\ P. O. Box 7062, Kampala, Uganda \\ ${ }^{2}$ Nabuin Zonal Agricultural Research and Development Institute, Uganda \\ ${ }^{3}$ National Crop Resources Research Institute Namulonge, P. O. Box 7084 Kampala, Uganda \\ ${ }^{4}$ National Agricultural Research Laboratories Institute, Kawanda, P. O. Box 7065 Kampala, Uganda \\ Corresponding author: haltalwana@ caes.mak.ac.ug
}

(Received 5 January, 2015; accepted 11 August, 2016)

\begin{abstract}
The bean bruchids, Acanthoscelides obtectus Say and Zabrotes subfasciatus Boheman (Coleoptera: Bruchidae), are cosmopolitan pests of stored dry common beans (Phaseolus vulgaris L.), causing damage through reduction of grain quality and seed germination. Biological resistance to these bruchids was definitively established in noncultivated bean accessions, and has been introgressed into a range of drybean market classes. However, existing resistance to bruchids in Uganda's common bean germplasm has not been systematically studied. In this study, 45 bean genotypes from the National Bean-Breeding Programme ( 25 genotypes) and agroecologically diverse bean growing areas in Uganda (20 genotypes), were evaluated for postharvest bruchid resistance. None of the evaluated bean genotypes expressed resistance to either bruchid species, with all the 45 bean genotypes supporting bruchid development, reproduction and feeding. All genotypes were severely damaged by bruchids feeding, resulting in significant $(\mathrm{P}<0.05)$ reduction of seed germination. Reduction in seed germination was related to the number of emergence holes and seed size; small bean seeds damaged by up to 2 bruchid emergence holes had a $7.1 \%$ reduction in germination, while large bean seeds with a similar number of emergence holes showed a $25 \%$ reduction in germination. Whereas this study further confirms bruchids as important storage pests of beans causing direct loss through consumption of the seed and indirect loss through viability deterioration, the resistance to bruchids in the evaluated range of Uganda's dry bean germplasm is inadequate for direct exploitation in a breeding programme.
\end{abstract}

Key Words: Acanthoscelides obtectus, Phaseolus vulgaris, Zabrotes subfasciatus

\section{RÉSUMÉ}

Les bruches du haricot, Acanthoscelides obtectus Say et Zabrotes subfasciatus Boheman (Coleoptera: Bruchidae), sont des ravageurs cosmopolites des haricots secs stockés (Phaseolus vulgaris L.), provoquant des dommages en réduisant la qualité des grains et le pouvoir germinatif des semences. La résistance biologique à ces bruches a été définitivement établie dans des lignées non cultivées de haricots et a été introgressée dans une gamme variée de haricots commercialisés. Cependant, la résistance actuelle aux bruches dans le matériel génétique des haricots communément répandus en Ouganda n'a pas été systématiquement étudiée. Dans la présente étude, 45 génotypes de haricots provenant du Programme national d'amélioration du haricot (25 génotypes) et de diverses zones agroécologiques de culture de haricots en Ouganda (20 génotypes), ont été évalués pour leur résistance post-récolte aux bruches. Aucun des génotypes évalués n'a exprimé de la résistance aux deux espèces de bruches, et tous les 45 génotypes ont favorisé le développement des bruches, leur reproduction et leur alimentation. Tous les 
génotypes ont été gravement endommagés du fait de l'alimentation des bruches, ce qui a entraîné une réduction significative $(\mathrm{P}<0,05)$ de la germination des graines. La réduction du pouvoir germinatif était liée au nombre de trous causés sur les graines et à la taille des graines; les petites graines de haricot endommagées avec jusqu'à 2 trous ont montré une réduction de 7,1\% de leur pouvoir germinatif, tandis que les grandes graines de haricots endommagées avec le même nombre de trous ont connu une réduction de $25 \%$ de pouvoir germinatif. Bien que cette étude confirme davantage que les bruches sont d'importants ravageurs en phase de stockage des haricots causant des pertes à la fois directe par la consommation de la graine et indirecte par la détérioration de la viabilité des semences, la résistance aux bruches dans la gamme étudiée du matériel génétique de haricots secs de l'Ouganda est insuffisante pour une exploitation directe dans un programme d'amélioration variétale.

Mots Clés: Acanthoscelides obtectus, Phaseolus vulgaris, Zabrotes subfasciatus

\section{INTRODUCTION}

The bean bruchids, Acanthoscelides obtectus Say and Zabrotes subfasciatus Boheman (Coleoptera: Bruchidae), are among the most important and widespread storage pests in all major dry common beans (Phaseolus vulgaris L.) growing regions world wide (Msolla and Misangu, 2002; Schmale et al., 2002; Nadir et al., 2005). These bruchid species have similar biology and coexist; with several adults of both $A$. obtectus and Z. subfasciatus infesting the same seed at the same time (Ware, 1988). They cause extensive grain weight and quality losses through their feeding (Giga et al., 1990; Nahdy, 1994), and product alterations such as reduction of nutritional and aesthetic value; alteration of cooking characteristics (Mulungu et al., 2007); and reduction in viability of bruchid-damaged seeds (Nahdy, 1990).

Losses in dry weight of bean grains due to bruchid damage, have been estimated at about $70 \%$, especially where post-harvest management is poor (Nahdy, 1990; Songa and Rono, 1998; Mulungu et al., 2007). Ultimately, bruchid damage results in significant grain price discounts (Mshili et al., 2011), and bruchid damage in storage forces farmers to sell beans within 2 - 3 months after harvest, so as not to incur total grain losses (Giga et al., 1992).

One possible approach to sustainable bruchid management is the exploitation of host-plant resistance, an effective, economical, and environment friendly method of pest control (Miklas et al., 2006). Genetic resistance to bruchids was discovered in wild bean accessions from Mexico (Schoonhoven et al., 1983), and introgressed into a range of dry bean market classes (Cardona et al., 1990). Potential of resistance as a method of control to reduce losses caused by bruchids in beans has been illustrated in the arcelin containing inbred RAZ bean lines that have consistently shown complete resistance for bruchids (Cardona et al., 1992; Misangu et al., 2001). However, the success of breeding to transfer this resistance to wider germplasms has been limited and there is considerable variation in susceptibility to bruchid attack among most cultivars grown. For Uganda's common dry bean germplasm, the presence and distribution of bruchid resistance has not been systematically studied. The objective of this study was to evaluate bean genotypes existing among farmers in agroecologically different bean growing areas in Uganda for resistance against post-harvest infestation and damage by bruchids (A. obtectus and Z. subfasciatus).

\section{MATERIALS AND METHODS}

Culturing of bruchids. This study was conducted during 2011/2012 at the National Crop Resources Research Institute (NaCRRI) Namulonge and the National Agricultural Research Laboratories Institute (NARLI); Kawanda in Uganda. Bruchids initially collected from farmers' stored beans during the bean genotype collection were mass reared under ambient conditions within a laboratory, on bean varieties K20 and NABE 6 which are highly susceptible to bruchids (Padgham et al., 1992).

In 1-litre polystyrene jars, $400 \mathrm{~g}$ of dry bean seeds of either variety K 20 or NABE- 6 were placed and infested with 150 - 200 unsexed A. obtectus or Z. subfasciatus. The jars were closed with lid tops that were perforated to allow aeration, but 
prevent bruchid escape. The bruchids were left to oviposit on the seeds for 10 days and, then removed by sieving each culture medium through a $3 \mathrm{~mm}$-mesh sieve.

The bean seeds were further incubated for 5 - 6 weeks. To ensure age uniformity, the first lot of emerged bruchids was sieved and discarded, and those that emerged $2-3$ days thereafter, were collected for use in the experiment. In order to maintain the source of bruchids, new stock cultures were initiated as soon as each new generation of adults emerged, following the same procedure above.

The infested grain was regularly replaced with fresh ones. Relative humidity and temperature of the room were recorded hourly, daily, using a data logger (Shenzhen Yuwen Sensor System Co., Ltd, China).

Bruchid progeny development periods, reproduction and bean damage. Newly-harvested grains of each of the 45 genotypes (Table 1) were sun-dried to a moisture content of less than $14 \%$, and then solarised to kill carry-over bruchid eggs and/or adults, using a technique described by Agona and Nahdy (1998). Fifty seeds of each genotype were randomly picked, weighed and placed in transparent glass jars. They were then infested with 20 adult bruchids ( 1 - 3 days old) in a 50:50 female:male ratio. Sexing of $Z$. subfasciatus was based on the size of the insects and the characteristic colour of the elytra. That of $A$. obtectus was based on morphological characteristics of the genitalia or the last abdominal segments (pygidium) (Allen et al., 1996).

All the jars were laid out on laboratory shelves at room temperature $\left(21.5-28^{\circ} \mathrm{C}\right)$, in a completely randomised design, with four replications. The bruchids were allowed to oviposit for 10 days before being discarded. Adult bruchid emergence was monitored daily and bruchids that emerged were sieved out using a coarse test sieve and counted. This was repeated until no more emergences were observed, indicating the end of the generation. The median bruchid development period (MDP) on each genotype was calculated at mid-period of oviposition (5 days from initial infestation time) to the emergence of 50\% of the first generation (Derera et al., 2010). The number of adult bruchids that emerged and median development period were combined to

TABLE 1. Common Uganda dry bean genotypes tested for resistance to Acanthoscelides obtectus and Zabrotes subfasciatus

\begin{tabular}{|c|c|c|}
\hline Breeding lines (7) & Improved commercial varieties (18) & Farmers varieties $(20)$ \\
\hline NARBL 40-3 (Black-2) & NABE 1 & Golden beans round \\
\hline NARBL 50-1 & NABE 2 & Small seeded cream \\
\hline NARBL 53-1 & NABE 3 & Mary meda \\
\hline NARBL 53-3 & NABE 4 & Yellow round \\
\hline NARBL-60 & NABE 5 & Bam \\
\hline NARBL 244-1(Black-1) & NABE 6 & Ocuc \\
\hline \multirow[t]{14}{*}{ NARBL-220 } & NABE 7C & Dark greyish spotted \\
\hline & NABE 8C & Golden beans oval \\
\hline & NABE 9C & Pinkish stripe oval \\
\hline & NABE 10C & Purple brown \\
\hline & NABE 11 & Jewe \\
\hline & NABE 12C & Cheparron \\
\hline & NABE 13 & Yellow long \\
\hline & NABE 14 & Yellow short \\
\hline & NABE 15 & Kaula \\
\hline & NABE 16 & Kigome \\
\hline & K131 & Gantagazose \\
\hline & K132 & Black big size \\
\hline & & Nambale short \\
\hline & & Kanyebwa \\
\hline
\end{tabular}


calculate the susceptibility index for each genotype, using Dobie's formula (Dobie, 1974):

Dobie's Susceptibility Index (SI)

$\frac{\log _{\theta}(\text { No. Adult emerged })}{\text { Median Development Period (MDP) }} X 100$

Based on the Dobie's susceptibility index, the bean genotypes were grouped into four categories as resistant: SI 0 - 3; moderately resistant: SI 4 - 7; susceptible: SI 8 - 10; highly susceptible: SI $\geq 11$ (Sulehrie et al., 2003). Additionally, grain weight loss, which is an economic loss indicator of bruchid feeding, was calculated as follows:

Grain weight loss $(\%)=$ $\left(\frac{\text { Initial grain weigh-Final grain weight }}{\text { Initial grain weight }}\right) \times 100$

Seed damage by the bruchids was estimated by counting the number of adult emergence holes per seed and the seeds with $0-1,2-3,4-5$ and > 5 emergent holes were grouped and counted.

Seed germination. Seeds of three bean varieties differing in seed size: NABE 5 (small), NABE 6 (medium) and NABE 11 (big), where small $=0.5$ $0.9 \mathrm{~cm}$ diameter, medium $=1.0-1.5 \mathrm{~cm}$ diameter, and large $=1.6-2.0 \mathrm{~cm}$ diameter (IPGRI, 2001), were evaluated for the effect of bruchid damage on bean seed germination. One hundred seeds of each variety was placed in $150 \mathrm{ml}$ polystyrene jars and infested with 60 (30 females and 30 males) 0-3 day-old adult $A$. obtectus that were allowed to mate and oviposit for seven days before being discarded. The jars were placed on laboratory shelves at ambient conditions (temperature $=21.5$ $-28^{\circ} \mathrm{C}$ and relative humidity $65-70 \%$ ) in a completely randomised experimental design, with four replications. Adult bruchid emergence was monitored daily and emerged bruchids were removed and discarded. The experiment was discontinued after five days when no more weevils emerged. Un-infested seeds of each variety were also stored for the same period, to act as a control.
At the end of the experiment, the seeds of each variety were combined (a total of 400 seeds from the four replications per variety). Each seed in the combined lot per variety was inspected for bruchid emergence holes and sorted into 11 categories based on the number of bruchid emergence holes on the seed, namely $0,1,2,3,4$, $5,6,7,8,9$ and $10+$ emergence holes per seed. Ten seeds from each of the eleven damage categories for each of the three varieties were picked randomly and planted into individual plates filled with sterilised moistened lake sand for 10 days to test their ability to germinate. Seedlings that had well-developed essential structures of the root system, shoot axis, cotyledons and terminal buds were considered "normal germination", and their proportion was recorded per variety

Data analysis. The data collected were subjected to analysis of variance, using the Generalised Linear Model (GLM) procedure of GenStat Version 13 statistical software (Lawes Agricultural Trust, 2010). Simple (Pearson) correlations between different parameters were explored. Further, Principal Component analysis was performed to estimate the important variable measured that had the greatest contribution to the variance observed in the data. The identified variable were then used to run regression analysis to estimate the relative performance of the different genotypes with respect to the two bruchid species, A. obtectus and Z. subfasciatus.

\section{RESULTS}

There were significant $(\mathrm{P}<0.05)$ differences in total number of $F_{1}$ progeny that emerged, number of days to the first adult bruchid to emerge, number of days for $50 \%$ of adult bruchids to emerge, number of days for the last adult bruchid to emerge, median development period and susceptibility index between bruchid species on a specific bean genotype (Table 2). The bean gentoypes supported higher reproduction of $Z$. subfasciatus than A. obtectus, based on absolute values of fecundity of both bruchid species on the different genotypes (Tables 3 and 4, Fig. 1). Landrace "Kanyebwa" and elite genotype "NABE 15" recorded the highest adult A. obtectus 
TABLE 2. Analysis of Variance of estimated emerged progeny, progeny development periods, $F_{1}$ emerged adult Acanthoscelides obtectus and Zabrotes subfasciatus populations, Susceptibility index, rain weight loss, and damage caused after incubation on 45 different common Ugandan bean genotypes for d" 100 days

\begin{tabular}{|c|c|c|c|c|c|c|}
\hline Source & $\mathrm{df}$ & $\begin{array}{l}\text { Total } \mathrm{F}_{1} \\
\text { Progeny } \\
\text { emerged }\end{array}$ & $\begin{array}{c}\text { Median } \\
\text { Development } \\
\text { period }\end{array}$ & $\begin{array}{c}\text { Seed } \\
\text { damage (\%) }\end{array}$ & $\begin{array}{l}\text { Grain } \\
\text { weight } \\
\text { loss (\%) }\end{array}$ & $\begin{array}{l}\text { Susceptibility } \\
\text { index }\end{array}$ \\
\hline Total & 89 & 7010.02 & 22.73 & 37.52 & 74.79 & 1.88 \\
\hline Bruchid species & 1 & $334567^{\star \star *}$ & $876^{\star * *}$ & $1462.09^{* * *}$ & $1254.34^{* * *}$ & $17.56^{\star \star}$ \\
\hline Bean genotype & 44 & $3759 \mathrm{~ns}$ & $13.03 \mathrm{~ns}$ & $21.72 \mathrm{~ns}$ & $75.63 \mathrm{~ns}$ & $1.79 \mathrm{~ns}$ \\
\hline Genotype x species & 44 & $2817^{\star * *}$ & $13.05^{\star \star *}$ & $20.95 n s$ & $47.13 n s$ & $1.61^{* \star \star}$ \\
\hline Error & & 1225.37 & 4.40 & 15.31 & 48.00 & 0.62 \\
\hline C.V $(\%)$ & & 21 & 4 & 9 & 45 & 7 \\
\hline \multicolumn{7}{|c|}{ Zabrotes subfasciatus } \\
\hline Bean genotype & 44 & 11953ns & $69.23^{*}$ & $30.59^{\star}$ & $374^{* *}$ & $4.732 \mathrm{~ns}$ \\
\hline \multicolumn{7}{|c|}{ Acanthoscelides obtectus } \\
\hline Bean genotype & 44 & $10024^{* *}$ & $29.84^{* *}$ & $129.67^{\star}$ & $154.07^{\star *}$ & $8.01^{*}$ \\
\hline
\end{tabular}

Values are mean squares; ${ }^{*}{ }^{* *},{ }^{* * *}=F$ value significant at $P<0.05,0.01$ and 0.001 , respectively; $n s=F$ value not significant at $P=0.05$

emergence; while landrace "Golden bean round" and breeding line NARBL 40-3 (Black-2) recorded the lowest adult $A$. obtectus emergence. On the other hand, landraces "Purple brown", "Yellow round", "Kigome", "Kaula", and "Gantagazose" recorded the highest adult Z. subfasciatus emergence; while landraces "Golden beans oval", "Bam" and "Mary Meda" recorded the lowest adult $Z$. subfasciatus emergence.

Mean development period for A. obtectus of was significantly $(\mathrm{P}<0.01)$ shorter than for $Z$. subfasciatus. However, the days to first emergence of adult bruchids were significantly $(\mathrm{P}<0.01)$ shorter for $\mathrm{Z}$. subfasciatus than $A$. obtectus. In contrast, the number of days to the last emergence of adult bruchids was significantly $(\mathrm{P}<0.01)$ longer for $\mathrm{Z}$. subfasciatus than $A$. obtectus (Tables 3 and 4). Based on the Dobie's Susceptibility Index all the bean genotypes evaluated were susceptible or highly susceptible to A. obtectus and Z. subfasciatus (Table 5).

All the evaluated genotypes were severely damaged by the bruchids (Tables 6 and 7). Seed damage was significantly $(\mathrm{P}<0.01)$ higher in $Z$. subfasciatus than A. obtectus infested seeds. Acanthoscelides obtectus damaged seeds were $77 \%$, of which seeds having $1-3$ holes constituted
$55 \%$ of the damage, while, Z. subfasciatus damaged seeds were $93 \%$, of which seeds having more than 4 holes contributed $58 \%$ of the damage. Bruchid damage resulted in varying degree of grain weight loss, being significantly $(\mathrm{P}<0.05)$ higher in Z. subfasciatus than in A. obtectus infested seeds.

Within each bruchid species, there were significant $(\mathrm{P}<0.01)$ differences in grain weight loss among the genotypes. In A. obtectus infested beans, grain weight loss was highest in Kayebwa and Nabe 15 (27\%) and least in NABE 4 (3.7\%), Golden brown - rounded (3.3\%), Dark grayish spotted (2.4\%) and NARBL 40-3 (Black 2) (2.3\%) (Table 6). In Z. subfasciatus infested seed, grain weight loss was highest in K131 and Yellow round (37\%) and the least in NABE 11 (4.4\%) (Table 7). Additionally, bruchid damage significantly $(\mathrm{P}<0.05)$ reduced germination in relation to number of emergence holes and seed size. Small bean seeds damaged by up to 2 bruchid emergence holes had small reduction in emergence and seedling vigour; while large bean seeds with similar number of emergence holes showed a significant reduction in germination (Fig. 2). 
TABLE 3. Estimated progeny development periods and $\mathrm{F}_{1}$ emerged adult populations of $A$ canthoscelides obtectus after incubation on 45 different common Ugandan bean genotypes for $<100$ days (Mean \pm SEM) ${ }^{*}$

\begin{tabular}{|c|c|c|c|c|c|}
\hline Bean genotype & $\begin{array}{l}\text { Total adult } \\
\text { weevils } \\
\text { emerged }\end{array}$ & $\begin{array}{l}\text { Days for first } \\
\text { adult weevil } \\
\text { to emerge }\end{array}$ & $\begin{array}{l}\text { Days for } 50 \% \\
\text { adult weevils } \\
\text { to emerge }\end{array}$ & $\begin{array}{l}\text { Days for last } \\
\text { adult weevil } \\
\text { to emerge }\end{array}$ & $\begin{array}{l}\text { Median } \\
\text { Development } \\
\text { period }\end{array}$ \\
\hline Kanyewa & $295.7 \pm 82.1$ & $34.0 \pm 0.0$ & $40.0 \pm 1.7$ & $57.0 \pm 3.1$ & $40.0 \pm 0.9$ \\
\hline NABE 15 & $243.7 \pm 65.5$ & $34.0 \pm 0.0$ & $41.3 \pm 0.3$ & $61.3 \pm 4.9$ & $41.9 \pm 0.7$ \\
\hline Gantagazose & $231.0 \pm 110.1$ & $34.0 \pm 0.0$ & $39.7 \pm 2.9$ & $53.0 \pm 3.5$ & $42.3 \pm 0.3$ \\
\hline NARBL 244 (Black 1) & $212.3 \pm 76.1$ & $34.0 \pm 0.0$ & $42.0 \pm 0.6$ & $57.0 \pm 3.2$ & $43.0 \pm 0.7$ \\
\hline Kigome & $164.0 \pm 43.9$ & $34.3 \pm 0.3$ & $39.3 \pm 1.8$ & $57.0 \pm 6.5$ & $42.7 \pm 0.5$ \\
\hline Golden Beans Oval & $160.7 \pm 76.4$ & $36.0 \pm 2.0$ & $42.3 \pm 0.3$ & $57.0 \pm 7.6$ & $44.1 \pm 2.0$ \\
\hline NABE 11 & $146.3 \pm 42.5$ & $35.4 \pm 0.5$ & $41.0 \pm 0.4$ & $54.0 \pm 2.2$ & $40.9 \pm 0.4$ \\
\hline Kaula & $146.0 \pm 40.7$ & $34.3 \pm 0.3$ & $40.7 \pm 0.7$ & $62.0 \pm 5.2$ & $42.6 \pm 0.4$ \\
\hline NABE 16 & $142.7 \pm 14.2$ & $34.0 \pm 0.0$ & $41.3 \pm 0.3$ & $61.3 \pm 6.9$ & $41.3 \pm 0.7$ \\
\hline K132 & $139.9 \pm 17.9$ & $37.0 \pm 1.1$ & $43.7 \pm 0.9$ & $57.3 \pm 2.0$ & $44.1 \pm 0.8$ \\
\hline Black Big Size & $132.7 \pm 32.2$ & $34.0 \pm 0.0$ & $37.7 \pm 2.7$ & $51.0 \pm 0.0$ & $41.0 \pm 0.8$ \\
\hline NARBL 220 & $132.3 \pm 87.1$ & $36.0 \pm 1.5$ & $42.0 \pm 1.2$ & $64.7 \pm 4.2$ & $45.2 \pm 2.7$ \\
\hline Nambale short & $130.3 \pm 23.4$ & $34.0 \pm 0.0$ & $38.7 \pm 1.5$ & $62.0 \pm 3.5$ & $38.9 \pm 0.7$ \\
\hline NABE 13 & $126.4 \pm 18.5$ & $38.0 \pm 1.4$ & $44.0 \pm 1.0$ & $55.4 \pm 1.1$ & $45.3 \pm 0.6$ \\
\hline NABE 14 & $126.4 \pm 33.9$ & $38.0 \pm 1.4$ & $43.6 \pm 1.6$ & $56.0 \pm 1.7$ & $45.0 \pm 0.9$ \\
\hline NARBL 60 & $113.3 \pm 59.7$ & $34.0 \pm 0.0$ & $42.0 \pm 0.0$ & $57.7 \pm 6.6$ & $40.5 \pm 2.2$ \\
\hline Yellow Long & $109.3 \pm 37.9$ & $34.0 \pm 0.0$ & $42.0 \pm 0.6$ & $54.3 \pm 3.3$ & $41.8 \pm 0.1$ \\
\hline NABE 1 & $107.1 \pm 24.5$ & $38.6 \pm 1.6$ & $44.7 \pm 0.8$ & $55.1 \pm 1.4$ & $45.5 \pm 0.8$ \\
\hline NABE $9 \mathrm{C}$ & $106.7 \pm 22.1$ & $36.9 \pm 1.1$ & $42.7 \pm 0.5$ & $57.0 \pm 1.4$ & $42.4 \pm 0.8$ \\
\hline Pinkish Stripe Oval & $106.7 \pm 56.8$ & $43.3 \pm 9.3$ & $47.0 \pm 7.6$ & $59.7 \pm 4.3$ & $58.1 \pm 16.9$ \\
\hline Purple Brown & $105.0 \pm 54.6$ & $34.7 \pm 0.3$ & $39.7 \pm 0.9$ & $61.3 \pm 5.8$ & $42.9 \pm 0.4$ \\
\hline Cheparron & $100.7 \pm 26.7$ & $34.7 \pm 0.3$ & $42.3 \pm 1.3$ & $53.3 \pm 3.0$ & $43.8 \pm 0.5$ \\
\hline Jewe & $99.7 \pm 44.1$ & $34.0 \pm 0.0$ & $39.7 \pm 2.4$ & $59.3 \pm 5.2$ & $42.8 \pm 1.3$ \\
\hline NARBL $53-3$ & $99.0 \pm 38.6$ & $35.3 \pm 1.3$ & $41.3 \pm 0.7$ & $50.3 \pm 5.5$ & $43.6 \pm 1.3$ \\
\hline NABE 5 & $97.9 \pm 12.5$ & $35.4 \pm 0.8$ & $41.9 \pm 0.3$ & $53.0 \pm 1.7$ & $42.7 \pm 0.3$ \\
\hline NABE $8 \mathrm{C}$ & $93.6 \pm 23.8$ & $37.1 \pm 1.1$ & $42.6 \pm 1.2$ & $56.3 \pm 2.3$ & $44.0 \pm 0.6$ \\
\hline NARBL $53-1$ & $89.7 \pm 36.2$ & $34.0 \pm 0.0$ & $40.0 \pm 0.6$ & $53.7 \pm 4.9$ & $41.6 \pm 1.0$ \\
\hline NABE 4 & $85.9 \pm 8.5$ & $38.4 \pm 1.4$ & $43.9 \pm 1.1$ & $59.1 \pm 2.4$ & $45.6 \pm 0.5$ \\
\hline Yellow Short & $85.0 \pm 33.1$ & $34.7 \pm 0.7$ & $41.3 \pm 2.0$ & $49.7 \pm 2.0$ & $43.5 \pm 1.7$ \\
\hline NABE 2 & $80.3 \pm 12.7$ & $37.9 \pm 1.3$ & $43.7 \pm 1.1$ & $55.9 \pm 2.5$ & $44.0 \pm 1.1$ \\
\hline NABE 3 & $79.6 \pm 21.6$ & $37.6 \pm 1.2$ & $43.9 \pm 0.6$ & $50.6 \pm 1.1$ & $43.9 \pm 0.6$ \\
\hline NABE $12 \mathrm{C}$ & $76.9 \pm 43.7$ & $39.3 \pm 1.9$ & $44.3 \pm 1.1$ & $52.7 \pm 2.0$ & $44.4 \pm 1.1$ \\
\hline NABE 6 & $75.0 \pm 18.6$ & $38.0 \pm 1.4$ & $43.6 \pm 1.2$ & $52.4 \pm 1.4$ & $44.4 \pm 0.8$ \\
\hline NABE $10 \mathrm{C}$ & $72.3 \pm 12.0$ & $38.6 \pm 1.6$ & $45.3 \pm 1.2$ & $57.1 \pm 3.1$ & $46.0 \pm 0.8$ \\
\hline NABE 7C & $68.7 \pm 18.5$ & $37.9 \pm 1.3$ & $43.3 \pm 1.4$ & $55.9 \pm 2.7$ & $45.3 \pm 0.8$ \\
\hline Ocuc & $65.3 \pm 14.3$ & $35.0 \pm 1.0$ & $40.3 \pm 0.7$ & $48.0 \pm 2.0$ & $41.9 \pm 1.8$ \\
\hline Dark Greyish Spotted & $64.7 \pm 17.7$ & $34.0 \pm 0.0$ & $39.3 \pm 2.2$ & $51.7 \pm 3.2$ & $40.0 \pm 1.2$ \\
\hline Bam & $60.7 \pm 25.3$ & $37.7 \pm 2.0$ & $49.0 \pm 6.6$ & $60.7 \pm 4.5$ & $47.4 \pm 3.9$ \\
\hline Yellow Round & $58.3 \pm 14.0$ & $34.7 \pm 0.3$ & $40.7 \pm 0.9$ & $53.3 \pm 5.9$ & $43.3 \pm 0.2$ \\
\hline K131 & $57.7 \pm 14.4$ & $37.9 \pm 1.4$ & $43.4 \pm 0.9$ & $57.6 \pm 2.5$ & $44.4 \pm 0.6$ \\
\hline NARBL $50-1$ & $57.3 \pm 32.3$ & $34.0 \pm 0.0$ & $40.3 \pm 1.2$ & $46.7 \pm 3.3$ & $39.8 \pm 1.6$ \\
\hline Mary Meda & $57.0 \pm 19.5$ & $34.0 \pm 0.0$ & $40.3 \pm 1.9$ & $58.3 \pm 3.8$ & $43.0 \pm 0.8$ \\
\hline Small Seeded Cream & $56.0 \pm 24.1$ & $36.7 \pm 2.2$ & $41.0 \pm 0.0$ & $53.0 \pm 3.6$ & $46.8 \pm 4.0$ \\
\hline Golden Beans Round & $37.3 \pm 12.4$ & $34.3 \pm 0.3$ & $39.7 \pm 1.9$ & $59.0 \pm 4.6$ & $40.1 \pm 1.9$ \\
\hline NARBL 40 - 3 (Black 2) & $37.3 \pm 16.7$ & $36.7 \pm 0.9$ & $40.7 \pm 1.2$ & $53.7 \pm 4.3$ & $47.0 \pm 0.8$ \\
\hline $\operatorname{LSD}(0.05)$ & 78.0 & 3.6 & 3.2 & 6.9 & 2.2 \\
\hline F probability & 0.002 & 0.044 & 0.007 & 0.128 & 0.004 \\
\hline
\end{tabular}

*sorted in descending order by total adult weevils emerged 
TABLE 4. Estimated progeny development periods and $F_{1}$ emerged adult populations of Zabrotes subfasciatus after incubation on 45 different common Ugandan bean genotypes for $<100$ days (Mean \pm SEM)*

\begin{tabular}{|c|c|c|c|c|c|}
\hline Bean genotype & $\begin{array}{c}\text { Total adult } \\
\text { weevils } \\
\text { emerged }\end{array}$ & $\begin{array}{l}\text { Days for first } \\
\text { adult weevil } \\
\text { to emerge }\end{array}$ & $\begin{array}{c}\text { Days for } 50 \% \\
\text { adult weevils } \\
\text { to emerge }\end{array}$ & $\begin{array}{l}\text { Days for last } \\
\text { adult weevil } \\
\text { to emerge }\end{array}$ & $\begin{array}{l}\text { Median } \\
\text { Development } \\
\text { period }\end{array}$ \\
\hline Purple Brown & $388.3 \pm 114.2$ & $28.3 \pm 0.9$ & $49.7 \pm 2.9$ & $65.7 \pm 1.5$ & $51.4 \pm 2.0$ \\
\hline Yellow Round & $348.7 \pm 20.7$ & $28.3 \pm 1.3$ & $46.0 \pm 1.2$ & $64.7 \pm 1.7$ & $49.7 \pm 1.0$ \\
\hline Kigome & $342.3 \pm 53.2$ & $28.3 \pm 1.3$ & $44.7 \pm 0.9$ & $61.7 \pm 3.4$ & $47.4 \pm 0.4$ \\
\hline Kaula & $323.0 \pm 92.4$ & $30.0 \pm 3.0$ & $47.3 \pm 3.3$ & $65.3 \pm 1.8$ & $51.8 \pm 5.9$ \\
\hline Gantagazose & $319.7 \pm 65.1$ & $29.3 \pm 1.9$ & $48.7 \pm 3.2$ & $63.3 \pm 1.8$ & $51.5 \pm 4.1$ \\
\hline NARBL $53-1$ & $312.7 \pm 140.3$ & $27.0 \pm 0.0$ & $47.0 \pm 3.5$ & $61.3 \pm 2.9$ & $46.4 \pm 2.4$ \\
\hline Nambale short & $299.7 \pm 72.8$ & $28.3 \pm 0.9$ & $49.7 \pm 2.8$ & $63.7 \pm 1.2$ & $51.4 \pm 3.1$ \\
\hline Cheparron & $282.0 \pm 62.1$ & $27.0 \pm 0.0$ & $46.7 \pm 2.7$ & $62.7 \pm 0.3$ & $46.7 \pm 1.0$ \\
\hline Dark Greyish Spotted & $280.0 \pm 146.3$ & $27.0 \pm 0.0$ & $49.7 \pm 3.2$ & $63.0 \pm 0.6$ & $49.5 \pm 2.0$ \\
\hline Kanyewa & $273.3 \pm 76.5$ & $30.3 \pm 1.8$ & $46.7 \pm 3.7$ & $61.0 \pm 3.2$ & $51.4 \pm 3.5$ \\
\hline K131 & $271.0 \pm 42.3$ & $33.9 \pm 1.9$ & $43.1 \pm 1.6$ & $56.7 \pm 2.4$ & $46.0 \pm 3.5$ \\
\hline Jewe & $264.7 \pm 80.7$ & $35.7 \pm 6.3$ & $49.0 \pm 3.0$ & $61.7 \pm 3.9$ & $58.1 \pm 8.1$ \\
\hline NABE 16 & $254.7 \pm 110.5$ & $27.0 \pm 0.0$ & $46.7 \pm 1.8$ & $61.0 \pm 2.6$ & $47.7 \pm 1.2$ \\
\hline NARBL 50 - 1 & $246.3 \pm 42.9$ & $27.0 \pm 0.0$ & $50.0 \pm 3.0$ & $63.3 \pm 0.7$ & $50.3 \pm 1.7$ \\
\hline NABE $8 \mathrm{C}$ & $246.0 \pm 30.2$ & $35.0 \pm 1.0$ & $43.4 \pm 1.9$ & $57.6 \pm 2.4$ & $47.2 \pm 3.6$ \\
\hline NABE 1 & $244.6 \pm 19.0$ & $33.0 \pm 1.3$ & $43.9 \pm 2.3$ & $59.7 \pm 2.3$ & $45.6 \pm 2.8$ \\
\hline NABE 5 & $242.1 \pm 46.9$ & $32.4 \pm 2.0$ & $43.4 \pm 1.4$ & $55.9 \pm 2.0$ & $43.9 \pm 1.4$ \\
\hline NARBL $53-3$ & $238.3 \pm 90.7$ & $27.0 \pm 0.0$ & $46.7 \pm 3.7$ & $61.7 \pm 2.0$ & $45.9 \pm 1.8$ \\
\hline NABE $9 \mathrm{C}$ & $236.0 \pm 32.1$ & $32.0 \pm 1.7$ & $43.3 \pm 1.9$ & $55.3 \pm 3.3$ & $44.2 \pm 1.9$ \\
\hline Pinkish Stripe Oval & $236.0 \pm 126.0$ & $31.7 \pm 3.7$ & $48.7 \pm 2.0$ & $64.3 \pm 0.9$ & $54.5 \pm 4.5$ \\
\hline NABE 2 & $229.9 \pm 33.9$ & $33.0 \pm 1.7$ & $43.6 \pm 2.0$ & $56.7 \pm 2.3$ & $44.6 \pm 1.7$ \\
\hline NABE 4 & $227.9 \pm 33.8$ & $32.7 \pm 1.6$ & $44.0 \pm 2.3$ & $56.4 \pm 3.3$ & $45.4 \pm 2.6$ \\
\hline NABE $10 \mathrm{C}$ & $227.0 \pm 27.0$ & $32.9 \pm 1.8$ & $45.0 \pm 2.3$ & $56.9 \pm 2.6$ & $45.8 \pm 2.2$ \\
\hline NABE 3 & $225.0 \pm 41.2$ & $32.1 \pm 1.8$ & $43.1 \pm 1.8$ & $54.0 \pm 3.0$ & $43.7 \pm 1.9$ \\
\hline NARBL 40-3 (Black 2) & $221.7 \pm 19.3$ & $32.7 \pm 3.0$ & $46.0 \pm 3.1$ & $63.3 \pm 1.2$ & $53.1 \pm 4.9$ \\
\hline NABE 6 & $216.3 \pm 40.9$ & $35.0 \pm 1.0$ & $43.9 \pm 2.1$ & $56.0 \pm 2.4$ & $47.8 \pm 4.0$ \\
\hline Yellow Short & $216.0 \pm 32.2$ & $29.0 \pm 2.0$ & $50.3 \pm 2.7$ & $64.0 \pm 0.6$ & $52.4 \pm 0.7$ \\
\hline NABE 13 & $211.7 \pm 40.4$ & $32.4 \pm 1.6$ & $44.3 \pm 2.3$ & $56.7 \pm 2.4$ & $45.3 \pm 2.4$ \\
\hline NABE 14 & $211.1 \pm 26.0$ & $32.0 \pm 1.8$ & $45.9 \pm 2.9$ & $56.9 \pm 3.0$ & $45.2 \pm 2.4$ \\
\hline NABE $12 \mathrm{C}$ & $204.9 \pm 28.2$ & $34.6 \pm 1.0$ & $45.1 \pm 2.3$ & $57.7 \pm 2.3$ & $47.8 \pm 3.2$ \\
\hline K132 & $204.1 \pm 26.2$ & $31.9 \pm 1.7$ & $45.7 \pm 2.5$ & $56.3 \pm 3.1$ & $44.7 \pm 2.1$ \\
\hline Yellow Long & $204.0 \pm 50.8$ & $29.0 \pm 1.0$ & $50.7 \pm 3.3$ & $63.7 \pm 1.2$ & $53.0 \pm 3.7$ \\
\hline NARBL 244 (Black 1) & $203.3 \pm 128.1$ & $37.0 \pm 1.0$ & $46.7 \pm 2.7$ & $61.7 \pm 1.5$ & $58.5 \pm 2.4$ \\
\hline NABE 15 & $202.7 \pm 62.5$ & $30.0 \pm 1.7$ & $50.0 \pm 2.1$ & $64.3 \pm 0.7$ & $52.9 \pm 1.7$ \\
\hline NABE $7 \mathrm{C}$ & $196.1 \pm 19.7$ & $33.3 \pm 1.7$ & $45.0 \pm 2.1$ & $56.1 \pm 2.1$ & $45.8 \pm 1.9$ \\
\hline NARBL 60 & $181.0 \pm 27.7$ & $27.7 \pm 0.7$ & $52.0 \pm 0.0$ & $64.0 \pm 1.2$ & $51.2 \pm 0.6$ \\
\hline Golden Beans Round & $180.0 \pm 32.9$ & $27.7 \pm 0.7$ & $53.0 \pm 0.6$ & $63.0 \pm 2.5$ & $53.1 \pm 1.5$ \\
\hline NABE 11 & $173.9 \pm 30.2$ & $34.4 \pm 0.8$ & $46.1 \pm 2.7$ & $57.6 \pm 2.8$ & $48.0 \pm 3.5$ \\
\hline Ocuc & $167.3 \pm 69.7$ & $29.7 \pm 2.7$ & $48.7 \pm 2.7$ & $64.0 \pm 2.1$ & $53.0 \pm 4.9$ \\
\hline Small Seeded Cream & $158.3 \pm 66.1$ & $30.7 \pm 3.7$ & $50.7 \pm 3.4$ & $61.3 \pm 2.3$ & $53.7 \pm 5.2$ \\
\hline Black Big Size & $155.7 \pm 71.1$ & $30.0 \pm 2.1$ & $52.3 \pm 0.3$ & $65.7 \pm 1.8$ & $54.2 \pm 3.0$ \\
\hline NARBL 220 & $154.3 \pm 56.5$ & $34.7 \pm 2.7$ & $52.7 \pm 1.3$ & $65.3 \pm 0.3$ & $59.4 \pm 4.5$ \\
\hline Golden Beans Oval & $150.3 \pm 84.3$ & $31.7 \pm 0.9$ & $48.7 \pm 2.9$ & $59.7 \pm 4.3$ & $54.2 \pm 2.3$ \\
\hline Bam & $127.7 \pm 53.7$ & $27.3 \pm 0.3$ & $49.3 \pm 3.3$ & $59.3 \pm 4.3$ & $49.1 \pm 2.8$ \\
\hline Mary Meda & $122.3 \pm 43.9$ & $31.7 \pm 4.7$ & $50.3 \pm 3.2$ & $61.7 \pm 3.5$ & $55.4 \pm 5.7$ \\
\hline $\operatorname{LSD}(0.05)$ & 115.8 & 4.3 & 5.7 & 6.3 & 7.1 \\
\hline F probability & 0.586 & 0.004 & 0.194 & 0.044 & 0.024 \\
\hline
\end{tabular}

*sorted in descending order by total adult weevils emerged 


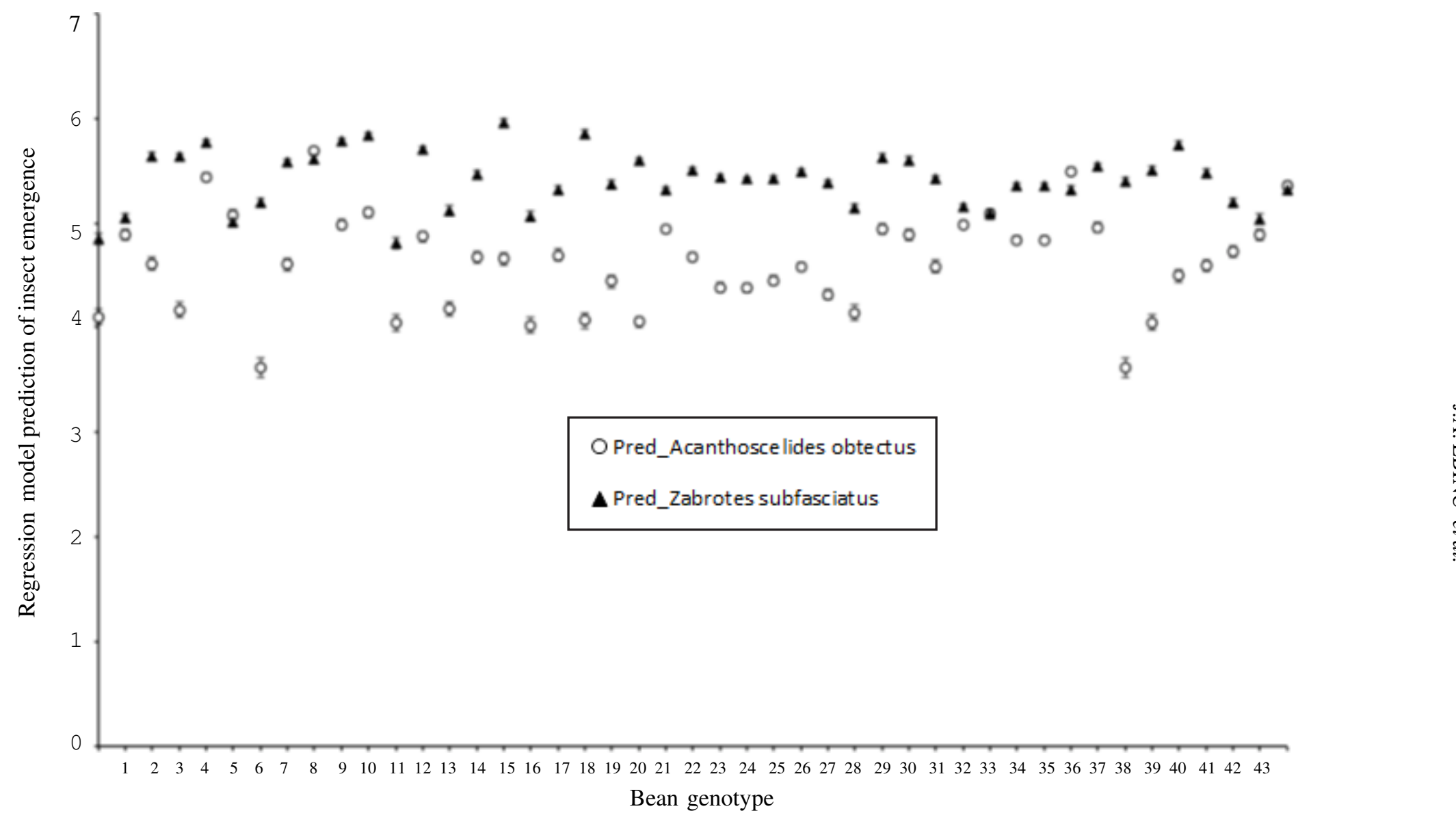

Figure 1. Scatter plot of total emerging $F_{1}$ progeny of Acanthoscelides obtectus and Zabrotes subfasciatus from 45 different genotypes as predicted from a generalized linear regression model using a Poisson distribution with logarithm link [Bean Genotypes: 1=Bam; 2="Black big size"; $3=$ Cheparron; 4="Dark greyish spotted"; $5=$ Gantagazose; $6="$ Golden beans oval"; $7="$ Golden beans round"; 8=Jewe; 9=Kanyewa; 10=Kaula; 11=Kigome; 12=Mary Meda; 13="Nambale short"; 14=Ocuc; 15="Pinkish stripe oval"; 16="Purple brown"; 17="Small seeded cream"; 18="Yellow long"; 19="Yellow

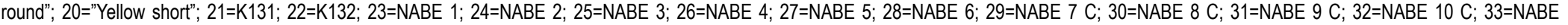
11; 34=NABE 12 C; 35=NABE 13; 36=NABE 14; 37=NABE 15; 38=NABE 16; 39=NARBL 40-3BLACK - 2; 40=NARBL 50 - 1; 41=NARBL 53 - 1; 42=NARBL 53 - 3; 43=NARBL - 60; 44=NARBL 220; 45=NARBL244-1BLACK-1]. 
TABLE 5. The susceptibility indices (Mean \pm SEM) and host reaction classes of 45 different common Ugandan bean genotypes evaluated for resistance after infestation with Acanthoscelides obtectus and Zabrotes subfasciatus (Mean \pm SEM) and incubating for d" 100 days*

\begin{tabular}{|c|c|c|c|c|}
\hline \multirow[t]{2}{*}{ Bean genotype } & \multicolumn{2}{|c|}{ Zabrotes subfasciatus } & \multicolumn{2}{|c|}{ Acanthoscelides obtectus } \\
\hline & Susceptibility index & Resistance class & Susceptibility index & Resistance class \\
\hline Kigome & $13.0 \pm 0.1$ & HS & $12.7 \pm 0.4$ & HS \\
\hline K131 & $12.9 \pm 0.5$ & HS & $8.7 \pm 0.8$ & $S$ \\
\hline NABE 1 & $12.7 \pm 0.7$ & HS & $10.1 \pm 0.5$ & HS \\
\hline NABE $8 \mathrm{C}$ & $12.7 \pm 0.4$ & HS & $10.2 \pm 0.9$ & HS \\
\hline NABE $9 \mathrm{C}$ & $12.7 \pm 0.6$ & HS & $10.7 \pm 0.5$ & HS \\
\hline Yellow Round & $12.7 \pm 0.3$ & HS & $9.9 \pm 0.8$ & $S$ \\
\hline NABE 2 & $12.5 \pm 0.8$ & HS & $9.9 \pm 0.5$ & $\mathrm{~s}$ \\
\hline NABE 3 & $12.5 \pm 0.5$ & HS & $9.4 \pm 0.7$ & S \\
\hline NABE 4 & $12.4 \pm 0.7$ & HS & $10.1 \pm 0.3$ & HS \\
\hline NABE 5 & $12.4 \pm 0.2$ & HS & $10.9 \pm 0.3$ & HS \\
\hline Kaula & $12.2 \pm 1.3$ & HS & $12.1 \pm 0.4$ & HS \\
\hline Cheparron & $12.1 \pm 1.0$ & HS & $10.8 \pm 0.8$ & HS \\
\hline Kanyewa & $12.1 \pm 1.4$ & HS & $14.1 \pm 0.2$ & HS \\
\hline NABE $10 \mathrm{C}$ & $12.1 \pm 0.6$ & HS & $9.3 \pm 0.7$ & $S$ \\
\hline NABE 13 & $12.1 \pm 1.0$ & HS & $10.9 \pm 0.5$ & HS \\
\hline NABE 6 & $12.0 \pm 1.0$ & HS & $9.6 \pm 0.8$ & $S$ \\
\hline NARBL $53-1$ & $12.0 \pm 1.6$ & HS & $10.6 \pm 1.5$ & HS \\
\hline Gantagazose & $11.9 \pm 1.2$ & HS & $13.3 \pm 1.8$ & HS \\
\hline NABE $12 \mathrm{C}$ & $11.9 \pm 0.8$ & HS & $7.9 \pm 1.5$ & $S$ \\
\hline NABE 14 & $11.9 \pm 1.0$ & HS & $10.9 \pm 0.8$ & HS \\
\hline Purple Brown & $11.9 \pm 1.3$ & HS & $10.7 \pm 1.9$ & $S$ \\
\hline K132 & $11.8 \pm 0.8$ & HS & $11.2 \pm 0.3$ & HS \\
\hline NABE $7 C$ & $11.8 \pm 0.6$ & HS & $8.9 \pm 0.9$ & $S$ \\
\hline NARBL 40-3 (Black 2) & $11.8 \pm 0.9$ & HS & $7.8 \pm 2.3$ & $S$ \\
\hline NARBL $53-3$ & $11.6 \pm 1.7$ & HS & $10.4 \pm 1.3$ & HS \\
\hline Nambale Short & $11.5 \pm 1.2$ & HS & $12.6 \pm 1.0$ & HS \\
\hline NABE 16 & $11.4 \pm 0.7$ & HS & $12.0 \pm 0.3$ & $\mathrm{HS}$ \\
\hline NABE 11 & $11.3 \pm 1.0$ & HS & $11.5 \pm 0.7$ & HS \\
\hline Jewe & $11.2 \pm 0.3$ & HS & $10.4 \pm 1.5$ & HS \\
\hline Dark Greyish Spotted & $11.0 \pm 1.8$ & HS & $10.5 \pm 1.3$ & HS \\
\hline NARBL $50-1$ & $11.0 \pm 0.8$ & HS & $8.8 \pm 2.4$ & $S$ \\
\hline Yellow Short & $10.7 \pm 0.3$ & HS & $10.3 \pm 1.9$ & HS \\
\hline NARBL 244 (Black 1) & $10.6 \pm 1.5$ & HS & $12.3 \pm 1.2$ & HS \\
\hline Pinkish Stripe Oval & $10.6 \pm 0.6$ & HS & $9.2 \pm 3.5$ & $S$ \\
\hline Yellow Long & $10.5 \pm 0.8$ & HS & $10.8 \pm 0.9$ & HS \\
\hline NABE 15 & $10.4 \pm 0.4$ & HS & $13.1 \pm 0.8$ & HS \\
\hline Ocuc & $10.2 \pm 0.5$ & HS & $10.2 \pm 0.4$ & HS \\
\hline NARBL 60 & $9.9 \pm 0.3$ & $\mathrm{~S}$ & $10.2 \pm 1.9$ & HS \\
\hline Small Seeded Cream & $9.9 \pm 1.5$ & $\mathrm{~s}$ & $8.8 \pm 1.9$ & $\mathrm{~S}$ \\
\hline Golden Beans Round & $9.7 \pm 0.3$ & $S$ & $9.0 \pm 1.2$ & S \\
\hline Golden Beans Oval & $9.6 \pm 0.6$ & $S$ & $10.3 \pm 2.7$ & HS \\
\hline Bam & $9.5 \pm 0.8$ & S & $7.8 \pm 1.4$ & $S$ \\
\hline Black Big Size & $9.3 \pm 0.8$ & $\mathrm{~s}$ & $12.9 \pm 1.1$ & HS \\
\hline NARBL 220 & $9.2 \pm 1.1$ & $S$ & $10.6 \pm 1.9$ & HS \\
\hline Mary Meda & $9.1 \pm 0.4$ & S & $9.7 \pm 1.2$ & $S$ \\
\hline $\operatorname{LSD}(0.05)$ & 2.0 & & 2.4 & \\
\hline F probability & 0.100 & & 0.027 & \\
\hline
\end{tabular}

Resistant classes: SI $0.0-2.5$ = resistant (R); SI $2.6-5.0$ = moderately resistant (MR); SI $5.1-7.5$ = moderately susceptible (MS); SI $7.6-10.0=$ susceptible $(S)$ and $\mathrm{SI}>10=$ highly susceptible $(\mathrm{HS})$; ${ }^{*}$ sorted in descending order by susceptibility index of $Z$. subfasciatus 
TABLE 6. Grain damage of 45 different common Ugandan bean genotypes by Acanthoscelides obtectus after incubation for d" 100 days (Mean $\pm \mathrm{SEM})^{*}$

\begin{tabular}{|c|c|c|c|c|c|c|}
\hline \multirow[t]{2}{*}{ Bean genotype } & \multirow[t]{2}{*}{$\begin{array}{c}\text { Seed } \\
\text { damage (\%) }\end{array}$} & \multicolumn{4}{|c|}{$\begin{array}{c}\text { Severity of seed damage estimated by the proportion (\%) } \\
\text { of seeds with a number of exit holes }\end{array}$} & \multirow[t]{2}{*}{$\begin{array}{r}\text { Grain weigh } \\
\text { loss }(\%)\end{array}$} \\
\hline & & 1 hole & $2-3$ holes & $4-5$ holes & $>5$ holes & \\
\hline Kanyewa & $95.3 \pm 4.7$ & $16.2 \pm 8.7$ & $24.6 \pm 6.6$ & $26.4 \pm 3.8$ & $32.7 \pm 11.6$ & $27.3 \pm 6.4$ \\
\hline NABE 15 & $90.7 \pm 5.2$ & $20.9 \pm 16.6$ & $23.2 \pm 4.3$ & $25.8 \pm 13.1$ & $30.2 \pm 16.7$ & $26.6 \pm 10.4$ \\
\hline Golden Beans Oval & $79.3 \pm 20.7$ & $36.5 \pm 26.9$ & $16.8 \pm 3.2$ & $24.0 \pm 12.2$ & $22.7 \pm 11.6$ & $25.2 \pm 9.4$ \\
\hline NABE 11 & $83.3 \pm 2.4$ & $22.1 \pm 12.7$ & $19.4 \pm 4.1$ & $19.3 \pm 3.1$ & $39.1 \pm 19.1$ & $21.2 \pm 11.4$ \\
\hline NABE 6 & $82.7 \pm 1.8$ & $46.4 \pm 10.6$ & $26.0 \pm 5.2$ & $13.0 \pm 2.3$ & $14.7 \pm 4.5$ & $20.3 \pm 5.5$ \\
\hline Jewe & $83.3 \pm 7.0$ & $61.2 \pm 18.4$ & $24.1 \pm 11.0$ & $9.7 \pm 5.6$ & $5.0 \pm 3.7$ & $19.9 \pm 9.3$ \\
\hline Kigome & $93.3 \pm 6.7$ & $28.2 \pm 18.1$ & $25.8 \pm 3.0$ & $22.5 \pm 9.8$ & $23.5 \pm 15.5$ & $19.8 \pm 6.1$ \\
\hline Ocuc & $86.0 \pm 7.0$ & $64.2 \pm 6.2$ & $26.8 \pm 5.8$ & $9.0 \pm 8.0$ & $0.0 \pm 0.0$ & $19.4 \pm 5.7$ \\
\hline Cheparron & $72.7 \pm 8.7$ & $42.5 \pm 12.2$ & $33.7 \pm 8.9$ & $19.0 \pm 7.8$ & $4.7 \pm 3.3$ & $18.5 \pm 2.8$ \\
\hline NARBL 244 (Black 1) & $90.7 \pm 6.6$ & $31.6 \pm 18.4$ & $31.2 \pm 2.8$ & $13.3 \pm 5.6$ & $23.9 \pm 14.3$ & $17.1 \pm 6.4$ \\
\hline Gantagazose & $90.0 \pm 6.4$ & $24.5 \pm 4.7$ & $35.7 \pm 14.1$ & $14.9 \pm 1.6$ & $24.9 \pm 17.5$ & $16.9 \pm 9.5$ \\
\hline NABE 3 & $71.3 \pm 15.7$ & $52.0 \pm 12.3$ & $16.9 \pm 8.9$ & $20.7 \pm 4.6$ & $10.4 \pm 5.2$ & $16.6 \pm 5.3$ \\
\hline NABE 2 & $90.7 \pm 4.8$ & $47.1 \pm 20.8$ & $27.9 \pm 7.1$ & $19.4 \pm 11.5$ & $5.6 \pm 2.3$ & $14.9 \pm 2.3$ \\
\hline NABE 14 & $87.3 \pm 7.0$ & $27.0 \pm 13.7$ & $29.7 \pm 14.1$ & $17.3 \pm 6.6$ & $26.0 \pm 21.0$ & $13.5 \pm 6.1$ \\
\hline Bam & $72.0 \pm 12.7$ & $65.2 \pm 11.6$ & $25.5 \pm 7.8$ & $8.0 \pm 4.0$ & $1.4 \pm 1.4$ & $12.9 \pm 5.7$ \\
\hline NARBL 53 - 3 & $78.7 \pm 12.7$ & $50.9 \pm 18.6$ & $35.1 \pm 12.0$ & $6.0 \pm 3.1$ & $8.0 \pm 4.2$ & $12.7 \pm 1.4$ \\
\hline Purple Brown & $69.3 \pm 19.2$ & $56.7 \pm 18.1$ & $20.5 \pm 4.8$ & $9.2 \pm 4.6$ & $13.7 \pm 10.9$ & $12.5 \pm 4.8$ \\
\hline NABE 13 & $90.0 \pm 5.8$ & $26.2 \pm 5.1$ & $33.7 \pm 9.6$ & $17.3 \pm 1.8$ & $22.9 \pm 4.2$ & $12.0 \pm 1.5$ \\
\hline NABE 1 & $87.3 \pm 2.4$ & $45.6 \pm 18.2$ & $25.8 \pm 2.6$ & $16.2 \pm 12.8$ & $12.4 \pm 5.6$ & $11.3 \pm 3.7$ \\
\hline Nambale short & $82.7 \pm 11.9$ & $47.0 \pm 15.5$ & $18.6 \pm 9.1$ & $13.9 \pm 5.6$ & $20.5 \pm 6.8$ & $10.9 \pm 1.6$ \\
\hline Black Big Size & $83.3 \pm 9.8$ & $47.7 \pm 13.3$ & $23.2 \pm 4.5$ & $18.2 \pm 11.2$ & $11.0 \pm 6.9$ & $10.6 \pm 3.8$ \\
\hline Kaula & $84.0 \pm 8.0$ & $35.8 \pm 8.4$ & $30.4 \pm 4.7$ & $22.8 \pm 7.5$ & $11.0 \pm 4.2$ & $10.5 \pm 2.0$ \\
\hline NARBL 220 & $66.7 \pm 17.6$ & $48.1 \pm 21.3$ & $27.9 \pm 4.5$ & $12.7 \pm 12.7$ & $11.3 \pm 11.3$ & $10.2 \pm 5.9$ \\
\hline NABE 12C & $78.7 \pm 0.7$ & $38.4 \pm 18.0$ & $30.5 \pm 2.7$ & $14.4 \pm 6.1$ & $16.7 \pm 13.1$ & $9.9 \pm 8.1$ \\
\hline Yellow Long & $63.3 \pm 7.7$ & $45.7 \pm 15.5$ & $30.0 \pm 2.7$ & $13.1 \pm 6.7$ & $11.2 \pm 7.3$ & $9.5 \pm 2.1$ \\
\hline K131 & $72.7 \pm 7.3$ & $52.2 \pm 17.3$ & $18.3 \pm 9.3$ & $22.2 \pm 9.4$ & $7.3 \pm 4.3$ & $9.4 \pm 5.0$ \\
\hline NARBL 60 & $73.3 \pm 17.6$ & $51.5 \pm 27.0$ & $20.3 \pm 7.8$ & $19.8 \pm 16.2$ & $8.3 \pm 6.0$ & $9.4 \pm 5.6$ \\
\hline Yellow Short & $60.7 \pm 10.4$ & $54.5 \pm 18.5$ & $23.9 \pm 7.6$ & $15.9 \pm 10.7$ & $5.7 \pm 5.7$ & $9.4 \pm 6.1$ \\
\hline NABE 10C & $90.0 \pm 10.0$ & $41.7 \pm 10.4$ & $33.6 \pm 6.7$ & $18.1 \pm 7.2$ & $6.6 \pm 4.1$ & $8.9 \pm 1.8$ \\
\hline K132 & $86.7 \pm 6.7$ & $24.5 \pm 11.6$ & $33.3 \pm 9.8$ & $23.3 \pm 8.5$ & $18.8 \pm 3.4$ & $8.6 \pm 5.7$ \\
\hline NABE 8C & $83.3 \pm 1.8$ & $32.9 \pm 1.4$ & $28.5 \pm 6.8$ & $20.8 \pm 1.3$ & $17.9 \pm 6.2$ & $8.1 \pm 2.5$ \\
\hline Pinkish Stripe Oval & $65.3 \pm 10.7$ & $52.6 \pm 24.9$ & $19.3 \pm 9.8$ & $14.9 \pm 8.4$ & $13.2 \pm 8.5$ & $8.0 \pm 4.7$ \\
\hline NABE $7 \mathrm{C}$ & $68.7 \pm 5.9$ & $51.1 \pm 25.6$ & $26.1 \pm 13.5$ & $15.0 \pm 8.7$ & $7.8 \pm 7.8$ & $7.7 \pm 7.0$ \\
\hline NABE 5 & $76.0 \pm 6.1$ & $43.0 \pm 6.2$ & $34.5 \pm 1.9$ & $17.2 \pm 5.1$ & $5.3 \pm 0.5$ & $7.6 \pm 3.4$ \\
\hline NARBL 50 - 1 & $51.3 \pm 13.5$ & $74.1 \pm 15.2$ & $12.2 \pm 6.5$ & $3.7 \pm 3.7$ & $10.1 \pm 5.6$ & $6.7 \pm 3.4$ \\
\hline NARBL $53-1$ & $66.7 \pm 18.5$ & $51.8 \pm 21.6$ & $29.1 \pm 11.9$ & $14.3 \pm 7.3$ & $4.8 \pm 3.9$ & $6.7 \pm 3.3$ \\
\hline Mary Meda & $60.0 \pm 2.3$ & $62.4 \pm 14.1$ & $25.0 \pm 8.0$ & $10.3 \pm 5.2$ & $2.2 \pm 2.2$ & $6.2 \pm 3.1$ \\
\hline NABE 16 & $86.0 \pm 5.0$ & $30.7 \pm 13.2$ & $40.0 \pm 5.9$ & $17.4 \pm 7.0$ & $11.9 \pm 5.0$ & $6.2 \pm 3.0$ \\
\hline NABE 9C & $86.7 \pm 6.7$ & $38.3 \pm 11.2$ & $34.0 \pm 6.7$ & $15.5 \pm 1.0$ & $12.2 \pm 7.7$ & $6.0 \pm 3.4$ \\
\hline Yellow Round & $63.3 \pm 7.7$ & $61.2 \pm 14.2$ & $18.8 \pm 5.3$ & $10.7 \pm 4.5$ & $9.3 \pm 5.6$ & $6.0 \pm 1.8$ \\
\hline Small Seeded Cream & $62.7 \pm 15.3$ & $70.1 \pm 16.4$ & $16.2 \pm 8.4$ & $12.0 \pm 6.7$ & $1.7 \pm 1.7$ & $5.8 \pm 2.6$ \\
\hline NABE 4 & $72.7 \pm 8.5$ & $57.0 \pm 2.6$ & $33.1 \pm 5.2$ & $7.4 \pm 1.6$ & $2.5 \pm 1.5$ & $3.7 \pm 1.1$ \\
\hline Golden Beans Round & $57.3 \pm 1.3$ & $78.7 \pm 7.6$ & $20.2 \pm 8.7$ & $1.1 \pm 1.1$ & $0.0 \pm 0.0$ & $3.3 \pm 2.3$ \\
\hline Dark Greyish Spotted & $57.3 \pm 2.9$ & $60.7 \pm 14.2$ & $26.0 \pm 9.3$ & $9.9 \pm 8.0$ & $3.4 \pm 1.9$ & $2.4 \pm 1.3$ \\
\hline $\operatorname{LSD}(0.05)$ & 13.8 & 15.2 & 9.6 & 9.5 & 10.8 & 14.8 \\
\hline F probability & 0.010 & 0.204 & 0.649 & 0.775 & 0.012 & 0.007 \\
\hline
\end{tabular}

*sorted in descending order by Grain weight loss (\%) 
TABLE 7. Grain damage of 45 different common Ugandan bean genotypes by Zabrotes subfasciatus after incubation for d" 100 days (Mean \pm SEM)

\begin{tabular}{|c|c|c|c|c|c|c|}
\hline \multirow[t]{2}{*}{ Bean genotype } & \multirow[t]{2}{*}{$\begin{array}{c}\text { Seed } \\
\text { damage (\%) }\end{array}$} & \multicolumn{4}{|c|}{$\begin{array}{c}\text { Severity of seed damage estimated by the proportion (\%) } \\
\text { of seeds with a number of exit holes }\end{array}$} & \multirow[t]{2}{*}{$\begin{array}{l}\text { Grain weigh } \\
\text { loss }(\%)\end{array}$} \\
\hline & & 1 hole & $2-3$ holes & $4-5$ holes & $>5$ holes & \\
\hline K131 & $98.7 \pm 1.3$ & $0.0 \pm 0.0$ & $7.4 \pm 4.1$ & $48.9 \pm 17.7$ & $43.6 \pm 15.0$ & $37.2 \pm 8.7$ \\
\hline Yellow Round & $99.3 \pm 0.7$ & $4.8 \pm 4.8$ & $5.4 \pm 3.6$ & $40.9 \pm 3.1$ & $48.9 \pm 5.2$ & $37.1 \pm 14.9$ \\
\hline Purple Brown & $100.0 \pm 0.0$ & $0.7 \pm 0.7$ & $4.0 \pm 4.0$ & $36.0 \pm 16.0$ & $59.3 \pm 20.7$ & $33.8 \pm 12.2$ \\
\hline NABE 2 & $90.0 \pm 10.0$ & $13.3 \pm 13.3$ & $22.8 \pm 16.0$ & $26.6 \pm 10.8$ & $37.3 \pm 18.7$ & $33.0 \pm 14.5$ \\
\hline NABE 15 & $94.0 \pm 3.1$ & $5.2 \pm 5.2$ & $9.5 \pm 4.6$ & $42.9 \pm 6.2$ & $42.4 \pm 9.9$ & $32.9 \pm 6.7$ \\
\hline NABE 3 & $90.0 \pm 7.2$ & $10.2 \pm 8.2$ & $24.0 \pm 12.5$ & $32.3 \pm 14.3$ & $33.5 \pm 29.4$ & $32.1 \pm 9.5$ \\
\hline Jewe & $88.0 \pm 6.1$ & $20.5 \pm 11.6$ & $23.3 \pm 14.5$ & $22.0 \pm 6.8$ & $34.2 \pm 26.9$ & $29.8 \pm 12.0$ \\
\hline Yellow Short & $96.0 \pm 4.0$ & $8.8 \pm 2.8$ & $45.3 \pm 14.7$ & $29.3 \pm 10.5$ & $16.5 \pm 6.6$ & $29.8 \pm 9.5$ \\
\hline Golden Beans Round & $88.0 \pm 4.0$ & $11.2 \pm 4.5$ & $35.1 \pm 10.4$ & $40.4 \pm 11.3$ & $13.3 \pm 5.0$ & $29.6 \pm 10.1$ \\
\hline Cheparron & $94.7 \pm 5.3$ & $11.0 \pm 10.0$ & $15.8 \pm 9.7$ & $33.9 \pm 7.6$ & $39.3 \pm 23.1$ & $28.2 \pm 7.7$ \\
\hline Kigome & $98.0 \pm 2.0$ & $2.8 \pm 1.4$ & $15.2 \pm 5.3$ & $37.5 \pm 11.6$ & $44.6 \pm 15.7$ & $28.1 \pm 9.6$ \\
\hline NARBL $53-3$ & $84.7 \pm 8.7$ & $12.4 \pm 8.5$ & $25.6 \pm 11.9$ & $30.1 \pm 7.2$ & $32.0 \pm 14.5$ & $25.8 \pm 12.3$ \\
\hline Golden Beans Oval & $87.3 \pm 6.6$ & $21.9 \pm 9.0$ & $28.6 \pm 10.9$ & $25.0 \pm 7.7$ & $24.5 \pm 14.8$ & $25.1 \pm 3.5$ \\
\hline NABE 5 & $100.0 \pm 0.0$ & $2.0 \pm 2.0$ & $18.0 \pm 13.1$ & $28.0 \pm 7.6$ & $52.0 \pm 8.1$ & $24.7 \pm 5.9$ \\
\hline NABE 6 & $93.3 \pm 4.8$ & $21.6 \pm 15.8$ & $30.9 \pm 15.8$ & $16.0 \pm 4.7$ & $31.5 \pm 25.5$ & $24.1 \pm 11.9$ \\
\hline Kaula & $94.7 \pm 5.3$ & $7.4 \pm 4.1$ & $7.6 \pm 3.2$ & $51.3 \pm 18.1$ & $33.7 \pm 23.7$ & $23.1 \pm 7.4$ \\
\hline NARBL $53-1$ & $99.3 \pm 0.7$ & $2.7 \pm 1.3$ & $18.8 \pm 8.7$ & $49.8 \pm 9.3$ & $28.8 \pm 17.7$ & $22.3 \pm 13.3$ \\
\hline Kanyewa & $95.3 \pm 4.7$ & $3.1 \pm 3.1$ & $18.1 \pm 13.1$ & $26.2 \pm 7.1$ & $52.7 \pm 20.2$ & $21.9 \pm 14.3$ \\
\hline Ocuc & $90.0 \pm 5.0$ & $19.8 \pm 18.7$ & $20.6 \pm 5.5$ & $48.1 \pm 17.4$ & $11.4 \pm 5.7$ & $21.9 \pm 7.7$ \\
\hline NABE $8 \mathrm{C}$ & $96.7 \pm 3.3$ & $1.3 \pm 0.7$ & $8.2 \pm 1.9$ & $70.5 \pm 3.4$ & $19.9 \pm 4.2$ & $21.4 \pm 9.6$ \\
\hline NARBL 60 & $75.3 \pm 13.1$ & $45.5 \pm 15.9$ & $24.4 \pm 8.8$ & $25.5 \pm 8.2$ & $4.6 \pm 2.3$ & $19.6 \pm 6.7$ \\
\hline Dark Greyish Spotted & $100.0 \pm 0.0$ & $1.3 \pm 1.3$ & $24.7 \pm 19.0$ & $28.7 \pm 18.8$ & $45.3 \pm 27.6$ & $17.9 \pm 12.5$ \\
\hline NARBL 50 - 1 & $95.3 \pm 4.7$ & $4.2 \pm 2.3$ & $10.7 \pm 1.7$ & $52.4 \pm 12.2$ & $32.8 \pm 15.6$ & $16.9 \pm 1.0$ \\
\hline NABE $7 C$ & $82.0 \pm 3.5$ & $22.7 \pm 13.9$ & $38.5 \pm 17.3$ & $25.3 \pm 4.3$ & $13.5 \pm 4.1$ & $16.8 \pm 3.1$ \\
\hline NABE 14 & $90.0 \pm 5.0$ & $21.2 \pm 15.2$ & $39.3 \pm 6.4$ & $24.1 \pm 4.7$ & $15.4 \pm 9.3$ & $15.8 \pm 7.5$ \\
\hline NABE $10 \mathrm{C}$ & $95.3 \pm 4.7$ & $13.7 \pm 11.8$ & $23.8 \pm 17.2$ & $53.6 \pm 10.7$ & $8.9 \pm 6.7$ & $15.1 \pm 8.0$ \\
\hline Nambale short & $100.0 \pm 0.0$ & $5.3 \pm 3.5$ & $20.7 \pm 10.7$ & $24.7 \pm 3.7$ & $49.3 \pm 14.4$ & $14.4 \pm 3.2$ \\
\hline NARBL 40-3 (Black 2) & $92.0 \pm 4.0$ & $6.1 \pm 3.0$ & $23.8 \pm 1.7$ & $50.5 \pm 11.2$ & $19.6 \pm 9.9$ & $14.3 \pm 2.8$ \\
\hline Bam & $93.3 \pm 6.7$ & $20.2 \pm 6.2$ & $36.3 \pm 4.1$ & $37.8 \pm 7.3$ & $5.7 \pm 1.2$ & $14.0 \pm 7.6$ \\
\hline Gantagazose & $100.0 \pm 0.0$ & $0.0 \pm 0.0$ & $15.3 \pm 7.0$ & $56.0 \pm 14.1$ & $28.7 \pm 15.8$ & $13.4 \pm 3.1$ \\
\hline Pinkish Stripe Oval & $98.0 \pm 2.0$ & $8.9 \pm 3.5$ & $19.4 \pm 8.6$ & $56.9 \pm 7.1$ & $14.8 \pm 3.4$ & $13.3 \pm 5.8$ \\
\hline NABE 16 & $90.0 \pm 5.3$ & $23.1 \pm 14.1$ & $25.3 \pm 13.6$ & $19.3 \pm 7.3$ & $32.3 \pm 21.1$ & $13.1 \pm 7.3$ \\
\hline NABE 4 & $92.7 \pm 7.3$ & $7.7 \pm 7.7$ & $25.3 \pm 15.2$ & $33.0 \pm 5.0$ & $34.0 \pm 17.8$ & $12.4 \pm 6.2$ \\
\hline NABE $9 \mathrm{C}$ & $100.0 \pm 0.0$ & $3.3 \pm 1.8$ & $21.3 \pm 15.4$ & $32.7 \pm 20.1$ & $42.7 \pm 23.7$ & $11.9 \pm 7.3$ \\
\hline Small Seeded Cream & $86.0 \pm 0.0$ & $31.0 \pm 12.1$ & $41.1 \pm 13.5$ & $8.5 \pm 3.4$ & $19.4 \pm 19.4$ & $11.7 \pm 4.7$ \\
\hline Mary Meda & $85.3 \pm 5.8$ & $26.7 \pm 11.9$ & $45.3 \pm 14.2$ & $25.2 \pm 15.8$ & $2.8 \pm 2.8$ & $11.1 \pm 4.9$ \\
\hline K132 & $86.7 \pm 6.8$ & $13.3 \pm 6.8$ & $39.4 \pm 15.7$ & $21.6 \pm 4.4$ & $25.7 \pm 18.2$ & $11.0 \pm 8.9$ \\
\hline NABE 1 & $91.3 \pm 4.4$ & $7.6 \pm 4.0$ & $36.9 \pm 12.6$ & $32.6 \pm 18.5$ & $22.8 \pm 10.8$ & $11.0 \pm 1.7$ \\
\hline Yellow Long & $96.7 \pm 3.3$ & $13.2 \pm 11.2$ & $29.9 \pm 4.1$ & $30.3 \pm 10.8$ & $26.7 \pm 5.7$ & $11.0 \pm 3.2$ \\
\hline NARBL 244 (Black 1) & $89.3 \pm 7.1$ & $19.5 \pm 10.7$ & $39.6 \pm 19.9$ & $26.8 \pm 20.7$ & $14.1 \pm 8.0$ & $9.6 \pm 7.5$ \\
\hline NABE 13 & $94.0 \pm 6.0$ & $15.2 \pm 9.2$ & $33.1 \pm 15.6$ & $20.4 \pm 1.6$ & $31.3 \pm 22.9$ & $9.2 \pm 6.8$ \\
\hline NARBL 220 & $90.7 \pm 1.8$ & $17.1 \pm 10.1$ & $37.6 \pm 18.3$ & $28.6 \pm 20.7$ & $16.7 \pm 8.4$ & $6.8 \pm 3.1$ \\
\hline NABE $12 \mathrm{C}$ & $90.7 \pm 4.8$ & $9.2 \pm 5.3$ & $46.1 \pm 12.1$ & $27.7 \pm 9.1$ & $16.9 \pm 8.0$ & $6.1 \pm 2.6$ \\
\hline Black Big Size & $97.3 \pm 2.7$ & $12.8 \pm 9.1$ & $39.6 \pm 10.7$ & $36.1 \pm 13.6$ & $11.5 \pm 3.6$ & $5.8 \pm 2.2$ \\
\hline NABE 11 & $90.0 \pm 5.0$ & $27.6 \pm 17.5$ & $30.0 \pm 14.5$ & $36.0 \pm 20.4$ & $6.4 \pm 5.4$ & $4.4 \pm 2.1$ \\
\hline $\operatorname{LSD}(0.05)$ & 7.1 & 10.4 & 14.9 & 16.5 & 21.7 & 23.2 \\
\hline F probability & 0.027 & 0.045 & 0.113 & 0.012 & 0.210 & 0.006 \\
\hline
\end{tabular}

*sorted in descending order by Grain weight loss (\%) 


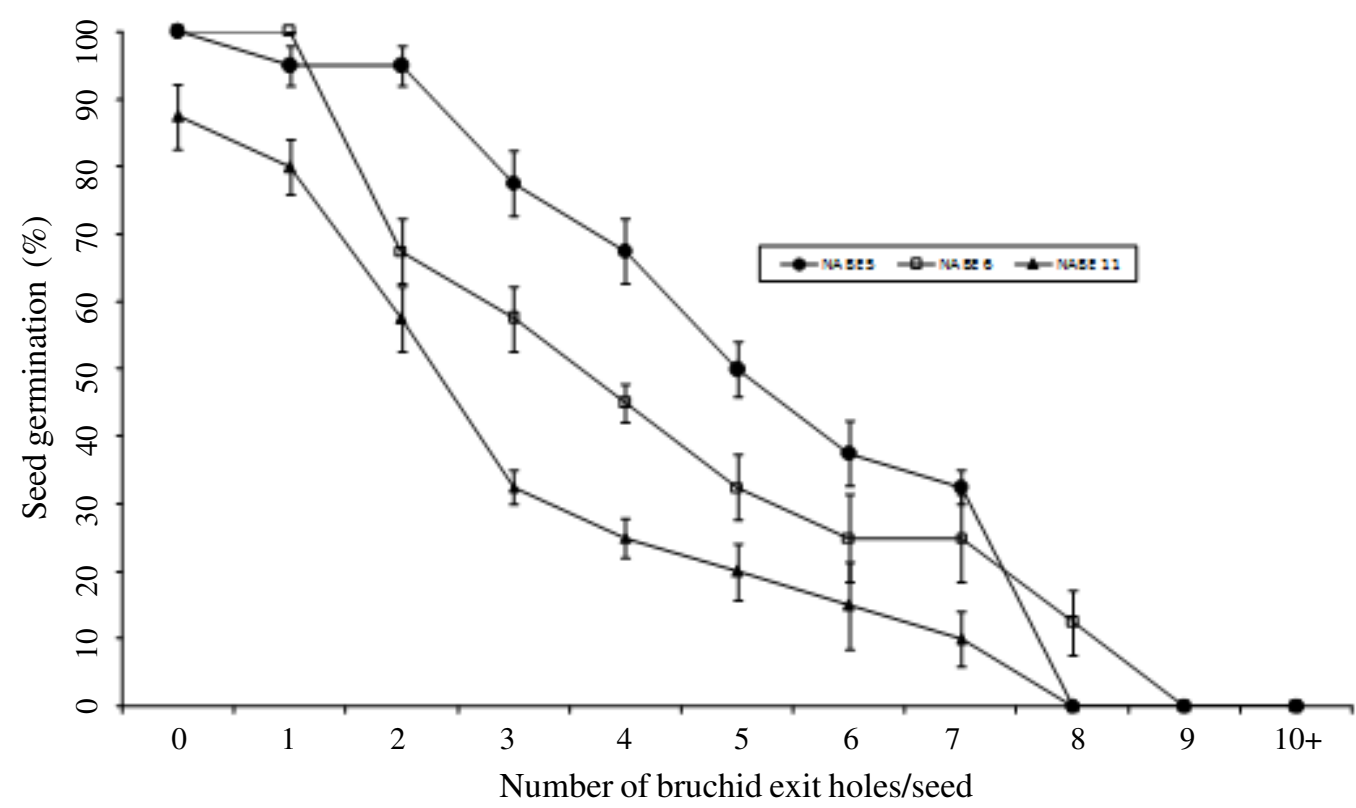

Figure 2. Effect of Acanthoscelides obtectus damage on seed germination of three bean varieties differing in seed size: NABE 5 (small), NABE 6 (medium) and NABE 11 (large).

\section{DISCUSSION}

The data presented clearly shows that all the 45 dry bean genotypes evaluated supported reproduction, growth and development of $A$. obtectus and Z. subfasciatus (Tables 3 and 4), thus providing evidence of susceptibility of all materials to the two-bruchid species. This indicates that the discovery of bruchid resistance in Mexico has not yet been tapped into Uganda's common bean germplasm. However, the genotypes were more susceptible to $Z$. subfasciatus than A. obtectus, and supported higher reproduction of $Z$. subfasciatus than $A$. obtectus. The warm temperatures in the laboratory in which the study was conducted (mean daily maximum temperature $27^{\circ} \mathrm{C}$ and mean daily minmum temperature $17^{\circ} \mathrm{C}$ ) may have favoured Z. subfasciatus, which prefers low altitude warmer areas (Schoonhoven and Cardona, 1986; Cardona, 1989; Cardona and Karel, 1990). On the other hand, the higher rate of multiplication of $Z$. subfasciatus than A. obtectus may be explained by the differences in preferential mode of bruchid species infestation, and egg oviposition. Zabrotes subfasciatus infestation and damage only occurs in the storage, starting from pre-existing insect populations and gluing eggs to the seed (Cardona et al., 1989; 1990; Cardona and Kornegay, 1999). This gives it a better adaptation to storage conditions than A. obtectus, whose infestation may begin from the standing crop in the field, by females ovipositing eggs on growing pods; while in storage A. obtectus scatters eggs among seeds (Shade et al., 1987; Acosta et al., 1992). Similarly, Z. subfasciatus progeny generally took longer (34-44 days) to emerge and develop than $A$. obtectus (27-37 days).

The average developmental period (Tables 3 and 4) was similar to that reported by Dendy and Credland (1991) and Parsons and Credland (2003), but the wide range of development times for both bruchid species would indicate differences in geographically distinct bruchid populations used in the evaluation. This, moreover, highlights that caution should be taken to compare pest status of these two species under the same physical conditions (Credland, 1994), and in making predictions about the responses of the different populations to new bean cultivars for agricultural use. This is so because each species (and 
biotype) may differ in its ability to infest or attack the same variety under different environmental conditions (Singh and Schwartz, 2011).

Resistance to bruchids in the range of dry bean market class varieties, breeding lines and landraces in Uganda evaluated in this study is inadequate, and limits direct exploitation of this germplasm. This further highlights the limited success of breeding to transfer bruchid resistance to wider germplasms, despite the discovery of resistance in non cultivated bean accessions three decades ago (Schoonhoven et al., 1983) and the rarity of natural occurrence of resistance to most of the storage insect pests in cultivated species, and instead suggests continued screening of very large numbers of drybean landraces and/or wild races.

Grain weight loss observed in the current study (Tables 6 and 7) is within range of losses due to bruchids reported in Africa (Karel and Autrique, 1989), and confirms that $Z$. subfasciatus and A. obtectus cause large losses in stored beans directly through their consumption of the seed. Additional loss due to bruchid damage is experienced through reduction in seed germination when damaged bean seeds are planted and either rot before germination or germinate into abnormal seedlings. Bruchid effect on bean seed germination is possibly due to damage to the embryo (Baier and Webster, 1992) or loss of food reserves a result of bruchid consumption of cotyledons (Mariga et al., 1993).

Although a farmer may plant small-seeded bean varieties with 1 - 2 bruchid emergence holes and experience minimal reduction in germination (Fig. 2), there is a significant reduction in germination of large seeded varieties even with 1 - 2 bruchid emergence holes. However, any bean seed with more than 3 emergence holes is probably not worth planting out or should be planted at a higher seeding rate with the expectation of lower emergence.

\section{CONCLUSION}

Evidently, Z. subfasciatus and A. obtectus are responsible for large post-harvest losses to beans directly through their consumption of the seed and, indirectly through the qualitative deterioration of the beans (and subsequently market value) or the reduced seed viability. Their infestation therefore is a menace to utilisation, storage and sustainability of production of the beans. With the inadequacy of resistance within the existing germplasm in Uganda, and the inherent restrictions to the use of pesticides, alternative integrated pest management options for bruchid control should urgently be sought.

\section{ACKNOWLEDGEMENT}

The Beans Programme of National Crops Resources Research Institute (NaCRRI) and the Postharvest Programme of the National Agricultural Research Laboratories Research Institute (NARLI) of the National Agricultural Research Organization (NARO), Uganda, are acknowledged for providing the bean genotypes and laboratory space for conducting the study. Dr. Thomas Odong of School of Agricultural Sciences Makerere University provided guidance with statistical analysis.

\section{REFERENCES}

Acosta, J., Rosales, R. and Garza, R. 1992. Resistance to the pod weevil in wild Phaseolus vulgaris. Annual Report of the Bean Improvement Cooperative 35:103 - 104. Agona, J.A. and Nahdy, S. M. 1998. Effect of solar drying period of beans on the viability, cooking time and injuriness of Acanthoscelides obtectus (Say). African Crop Science Journal 6:417 - 421.

Allen, D. J., Ampofo, J. K. O. and Wortrmann, C. S. 1996. Storage pests: Bruchids. In: Pests, Diseases, and Nutritional disorders of the Common bean in Africa: A field Guide. International Centre for Tropical Agriculture, Cali-Colombia. CIAT Publication Series No. 260. 132p.

Baier, A. H. and B. D. Webster. 1992. Control of Acanthoscelides obtectus Say (Coleoptera: Bruchidae) in Phaseolus vulgaris L. Seed stored on small farms - II. Germination and cooking time. Journal of Stored Products Research 28(4): 295-299.

Cardona, C. 1989. Insects and other invertebrate pests in Latin America and their control. In: Schwartz, H. F. and Pastor-Corrales, M.A. 
(Eds.). Bean production problems in the tropics. $2^{\text {nd }}$ Edition. Centro Internacional de Agricultura Tropical (CIAT), Cali, Colombia. pp. 505-571.

Cardona, C. and Karel, A.K. 1990. Key insects and other invertebrate pests of beans. In: Singh, S.R. (Ed.). Insect pests of tropical food legumes. John Wiley and Sons. Chichester. pp. $157-191$.

Cardona, C. and Kornegay, J. 1999. Bean germplasm resources for insect resistance. In Clement, S. L. and Quisenberry, S. S. (Eds.). Global plant genetic resources for insect resistance. CRC Press, Boca Raton, FL. pp. 85-99.

Cardona, C., Kornegay, J., Posso, C. E., Morales, F. and Ramírez, H. 1990. Comparative value of four arcelin variants in the development of dry bean lines resistant to the Mexican bean weevil. Entomologia Experimentalis et Applicata 56: 197-206.

Cardona, C., Dick, K., Posso, C.E., Ampofo, K. and Nadhy, S.M. 1992. Resistance of a common bean (Phaseolus vulgaris L.) cultivar to post harvest infestation by Zabrotes subfasciatus (Boheman) (Coleoptera: Bruchidae). II. Storage tests. Tropical Pest Management 38: 173- 175.

Cardona, C., Posso, C. E., Kornegay, J. L., Valor, J. and Serrano, M. 1989. Antibiosis effects of wild dry bean accession on the Mexican bean weevil and the bean weevil (Coleoptera: Bruchidae). Journal of Economic Entomology 82:310 - 315.

Credland, P.F. 1994. Bioassays with bruchid beetles: Problems and (some) solutions. In: Highley, E. , Wright, E.J., Banks, H.J., Champ, B.R. (Eds.). Stored Product Protection, Proceedings of the 6th International Working Conference on Stored-Product Protection, Canberra, Australia, 17 - 23 April 1994. CAB International, Wallingford, United Kingdom. pp. 516 - 509.

Dendy, J. and Credland, P. F. 1991. Development, fecundity and egg dispersion of Zabrotes subfasciatus. Entomologia Experimentalis et Applicata 59: 9 - 17.

Derera, J., Pixley, K.V. and Giga, D.P. 2010. Appraisal of protocol for the rapid screening of maize genotypes for maize weevil resistance. African Entomology 18(1):8-16.

Dobie, P. 1974. The Laboratory assessment of the inherent susceptibility of maize varieties to post harvest infestation by Sitophilus zeamais Motsch. (Coleoptera: Curculionidae). Journal of Stored Product Research 10: 183 197

Giga, D. P., Ampofo, J. K. O., Nahdy M. S., Negasi, F., Nah'Imana, F. and Nchimbi, S. 1992. "Onfarm storage losses due to bean bruchids and farmers' control strategies". A report on a Travelling Workshop 1992. CIAT Occassional Publication Series No.8.

Giga, D., Nchimbi, S. and Slumpa, S. 1990. Bruchid Research in Southern Africa. In: Smithson, J.B. (Ed.), Progress in Improvement of Common Beans in Eastern and Southern Africa. Proceedings of the Nineth SUA/CRSP and SADC/CIAT Bean Research Workshop, Morogoro, Tanzania. pp. 228-236.

IPGRI. 2001. Descriptors for Phaseolus vulgaris.International Plant Genetic Resources Institute, Rome, Italy. 1 pp. vi + 45 pp. ISBN 92- 9043-468-6.

Karel, A.K. and Autrique, A. 1989. Insects and other pests in Africa. pp. 455-504. In: Schwartz, H.F. and Pastor Corrales, M.A. (Eds.), Bean production problems in the tropics. 2nd Ed. CIAT, Cali, Colombia.

Lawes Agricultural Trust. 2010. GenStat $13^{\text {th }}$ edition release 13.1. GenStat Procedure Library Release, Lawes Agricultural Trust UK.

Mariga, I.K., Dube, R. and Munetsi, M. 1993. Effect of bruchid damage on the germination of grain legume. East African Journal of Agriculture and Forestry 58(3 - 4): 105-110

Miklas, P. N., Kelly, J.D., Beebe, S. E. and Blair, M.W. 2006. Common bean breeding for resistance against biotic and abiotic stresses: From classical to MAS breeding. Euphytica 147: 105-131.

Misangu, R.N., Nchimbi-Msolla, S. and Reuben, S.O.W.M. 2001. Resistance of arcelin incorporated bean (Phaseolus vulgaris L.) hybrids and their parental cultivars against the bean bruchid Zabrotes subfasciatus (Boh). Tanzania Journal of Agricultural Science 4: 23-28 
Mshili, F. J., Temu, A., Fulton, J. and LowenbergDeBoer, J. 2011. Consumer preferences as drivers of the common bean trade in Tanzania: A marketing perspective. Journal of International Food and Agribusiness Marketing 23:110-127.

Msolla, S.N. and Misangu, R.N. 2002. Seasonal distribution of common bean (Phaseolus vulgaris L.) bruchid species in selected areas in Tanzania. Proceeding Bean Seed Workshop, Arusha, Tanzania, 12-14 January 2002. pp. 1-5.

Mulungu, L.S., Luwondo, E.N., Reuben, S. and Misangu, R. 2007. Effectiveness of local botanicals as protectants of stored beans (Phaseolus vulgaris L.) against bean bruchid (Zabrotes subfasciatus Boh) (Genera: Zabrotes. Family: Bruchidae). Journal of Entomology 4: 210-217.

Nadir, A., Doyle, M., Martine, H.M., Celine, B., Leny, M. and Betty, B. 2005. Ancient and recent evolutionary history of the bruchid beetle, Acanthoscelides obtectus (Say), a cosmopolitan pest of beans. Molecular Ecology 14:1015-1024.

Nahdy, S.M. 1990. Distribution pattern of bean bruchids Zabrotes subfasciatus (Boh.) and Acanthoscelides obtectus (Say) (Coleoptera: Bruchidae) in Uganda. In: CIAT Proceedings of the second Workshop on beans research in Eastern Africa 5-8th March, Pp 273-281. CIAT African Workshop Series No. 7. Nairobi, Kenya.

Nahdy, S.M. 1994. Bean sieving, a possible control measure for the dried bean beetles, Acanthoscelides obtectus (Say) (Coleoptera: Bruchidae), Journal of Stored Products Research 30:65-69.

Parsons, D.J. and Credland, P.F. 2003. Determinants of oviposition in Acanthoscelides obtectus: A nonconformist bruchid. Physiological Entomology 28:221231.
Schmale, I., Wackers, F. L., Cardona, C. and Dorn, S. 2002. Field infestation of Phaseolus vulgaris by Acanthoscelides obtectus (Coleoptera: Bruchidae), parasitoid abundance, and consequences for storage pest control. Environmental Entomology 31:859-863.

Schoonhoven, A.V., Cardona, C. and Valor, J. 1983. Resistance to the bean weevil and Mexican Bean Weevil (Coleoptera: Bruchidae) in non cultivated bean accessions. Journal of Economic Entomology 76: 1255-1259.

Schoonhoven, V.A. and Cardona, C. 1986. Main insect pest of stored beans and their control. Study guide. Series 04EB-05-03. Centro Internaciaonal de Agricultura Tropical, Cali, Colombia. 40pp.

Shade, R.E., Pratt, R.C. and Pomeroy, M.A. 1987. Development and mortality of the bean weevil, Acanthoscelides obtectus (Coleoptera: Bruchidae) on mature seeds of tepary beans, Phaseolus acutifolius and common beans, Phaseolus vulgaris. Environmental Entomology 16:1067 - 1070.

Singh, S.P. and Schwartz, H.F. 2011. Review: Breeding common bean for resistance to insect pests and nematodes. Canadian Journal of Plant Sciences 91: 239 - 250.

Songa, J.M. and Rono, W. 1998. Indigenous methods for bruchid beetle (Coleoptera: Bruchidae) control in stored beans (Phaseolus vulgaris L.). International Journal of Pest Management 44:1 - 4.

Sulehrie, M.A.Q., Golob, P., Tran, B.M.D. and Farrell, G. 2003. The effect of attributes of Vigna spp. on the bionomics of Callosobruchus maculatus. Entomologia Experimentalis et Applicata 106:159 - 168.

Ware, G. W. 1988. Complete Guide to Pest Control: with and without Chemicals. Thomson Publications, Fresno. 388 pp. 